\title{
Délétions télomériques submicroscopiques et retard mental. Cas particulier du chromosome 16
}

Dans le groupe hétérogène des $\alpha$ thalassémies, un tableau extrêmement particulier était signalé en 1981 à propos de trois observations, associant la présence d'hémoglobinose $\mathrm{H}$ à un retard mental profond, sans qu'on puisse retrouver la transmission mendélienne habituelle. Les auteurs posaient la question d'un syndrome nouveau ou d'une étrange coïncidence [1]. L'étude attentive d'autres observations plus ou moins semblables a permis la dissociation de deux syndromes différents, tous deux appelés ATR (pour $\alpha$-thalassemia/mental retardation). Dans un premier groupe comportant uniquement des sujets mâles, aucune anomalie n'était retrouvée au niveau du locus $\alpha$-globine ; le retard mental très profond, global et précoce était accompagné de lésions dysmorphiques caractéristiques [2]. Des études familiales, enfin, permettaient de prouver une transmission liée au sexe, indépendante de celle des gènes $\alpha$-globine, dont le déterminant était localisé sur le chromosome X en Xq12-q21.31 [3] (m/s $n^{\circ} 1$, vol. 8, p. 73). Par opposition à ce syndrome ATR-X, l'observation d'un autre groupe de malades permettait la définition d'un syndrome ATR-16, dans lequel une symptomatologie neuro-psychiatrique variable était toujours accompagnée de grandes délétions (pouvant atteindre jusqu'à 1-2 mégabases, soit $\left.1-2.10^{6} \mathrm{pb}\right)$ du télomère du bras court du chromosome 16 et englobant le locus $\alpha$-globine [4]. Une première observation, où était démontrée la stabilisation du télomère par addition de l'hexamère caractéristi- que (TTAGGG) par une télomérase, a fait l'objet d'une nouvelle [5] $(\mathrm{m} / \mathrm{s}$ $n^{\circ} 10$, vol. 7, p. 1091); plusieurs autres cas ont été identifiés depuis. Le polymorphisme clinique s'en explique sans doute du fait que les délétions sont inégales en taille, et qu'elles s'accompagnent ou non de translocations submicroscopiques variables. Ces aneuploïdies complémentaires sont évidemment susceptibles d'avoir, elles aussi, une expression clinique. La description a, par exemple, été faite d'un enfant chez lequel une monosomie du télomère 16p s'accompagnait d'une trisomie du télomère du chromosome 1 [6]. Une attention particulière a cependant été portée aux cas de monosomie pure, qui pourraient aider à localiser, à proximité du locus $\alpha$ globine, des gènes essentiels pour un développement normal.

C'est l'étude comparative de plusieurs cas de ce type qui permet aujourd'hui une avancée significative [7]. Dans certains cas, dont celui cité plus haut, la délétion du télomère $16 p$, variable dans sa limite centromérique, ne s'étend cependant que peu au-delà des gènes de la famille $\alpha$-globine. Ces sujets ont apparemment un développement intellectuel normal, et il semble donc que la zone du télomère distale par rapport à l' $\alpha$-globine n'est pas impliquée dans le développement mental.

Une monosomie pure par ATR-16 a, par ailleurs, été démontrée chez un sujet présentant un handicap mental profond $(\mathrm{QI}=53)$ en même temps qu'une hémoglobinose $\mathrm{H}$ [4]. Une analyse cytogénétique de forte résolution avait mis en évidence un raccourcissement discret du bras court du chromosome, non retrouvé chez les parents. Le complément des gènes $\alpha$ était normal chez le père $(\alpha \alpha / \alpha \alpha)$, la mère était porteuse d'un trait a-thalassémique transmis à l'enfant $(-\alpha / \alpha \alpha)$; il s'agissait donc d'un événement de novo. La délétion de $16 p 3.3$, stabilisée comme dans les observations précédentes par des séquences télomériques répétées, était dans ce cas beaucoup plus grande $(2 \mathrm{Mb})$ et incluait un large fragment centromérique par rapport à l' $\alpha$-globine (figure 1). C'est dans cette zone critique qu'il fallait chercher le(s) gène(s) impliqué(s) dans le développement mental.

En comparant ce cas fortement démonstratif à ceux qui ne s'accompagnent pas de déficit mental, on définit une région minimale d'environ $1.7 \mathrm{Mb}$. Une cartographie préliminaire par enzymes de restriction a montré que la quasi-totalité de cette zone est exceptionnellement riche en dinucléotides $\mathrm{CpG}$, habituellement associés à la présence de gènes, et, dans une première approximation, le segment pourrait comprendre plus de 80 gènes. Par ailleurs, on ne sait pas si l'expression phénotypique est celle d'un ou deux gènes ou, à l'inverse, due à une action cumulative. Seule, sans doute, la comparaison de cas différents devrait permettre une délimitation plus précise du déterminant en cause.

Dans le cas particulier du chromosome 16 , une anomalie chromosomique, submicroscopique et presque cryptique, a été identifiée parce que 


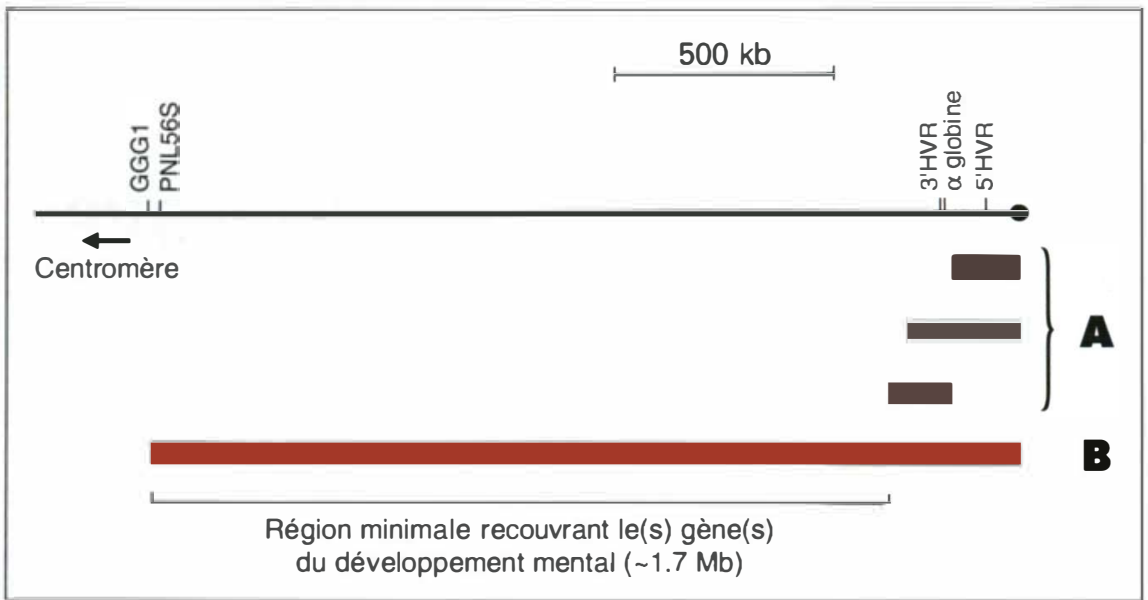

Figure 1. Représentation schématique de l'extrémité télomérique du bras court du chromosome 16. A proximité du télomère, le locus $\alpha$ globine, encadré par deux régions hypervariables (HVR). A. Extension de la délétion retrouvée chez différents sujets présentant un syndrome $\alpha$ thalassémique, mais pas de déficit intellectuel. B. Extension de la grande délétion submicroscopique chez le sujet présentant simultanément $\alpha$ thalassémie et retard mental. Les deux sondes (GGG1 et PNL56S) délimitent l'extrémité centromérique de cette délétion. La différence entre $A$ et $B$ définit la région minimale qui doit contenir le(s) gène(s) impliqué(s) dans le développement mental (d'après [7]).

l'attention avait été attirée par le phénotype particulier d'une $\alpha$ thalassémie et que l'on connaissait la localisation du locus $\alpha$-globine. Des processus génétiques analogues ont été observés au niveau d'autres télomères, mais toujours jusqu'ici parce qu'ils étaient associés à des phénotypes caractéristiques. La structure des télomères rend vraisemblable, dans d'autres chromosomes, l'existence d'événements submicroscopiques échappant à une étude cytogénétique de routine, qu'il s'agisse de délétion télomérique, de translocation, de disomie uniparentale. Les régions télomériques sont, en effet, particulièrement riches en gènes, mais aussi en séquences minisatellites hypervariables (HVP), qui facilitent les cassures. Un certain nombre de ces événements génétiques, qui restent souvent cryptiques, pourraient être à l'origine de retard mental. On estime, en effet, que $60 \%$ de ces retards sont d'origine génétique et plus de 500 dysfonctions monogéniques ont, par ailleurs, été décrites comme potentiel-
3. Gibbons RJ, Suthers (GK, Wilkie AOM, et al. X-linked $\alpha$-thalassemia/mental retardation (ATR-X) syndrome: localization to $\mathrm{Xq12-q21.31}$ by $\mathrm{X}$ inactivation and linkage analysis. Am J Hum Genet 1992 ; 51 : 1136-49. 4. Wilkie AOM, Buckle VJ, Harris PC, et al. Clinical features and molecular analysis of the $\alpha$ thalassemia/mental retardation syndrome. I. Cases due to deletions involving chromosome band 16p13.3. Am / Hum Genet 1990 ; 46: 1112-26.

5. Wilkie AOM, I amb J, Harris PC, et al. A truncated human chromosome 16 associated with $\alpha$ thalassaemia is stabilized by addition of telomeric repeat (TTAGGG)n. Nature 1990 ; 346 : 868-71

6. Iamb J, Harris PC, Lindenbaum RH, et al. Detection of breakpoints in submicroscopic chromosomal translocation illustrating an important mechanism for genetic disease. Lancet 1989; II : 819-24.

7. Lamb J, Harris PC, Wilkie AOM, et al. De novo ununcation of chromosome $16 \mathrm{p}$ and healing with (TTAGGG)n in the $\alpha$ thalassemia/mental retardation syndrome (ATR-16). Am J Hum Genet 1993; 52: 668-76. 8. Wahlstrom J. Gene map of mental retardation. I Meni Defic Res 1990; 34: 11-27.

9. Wilkie AOM. Detection of cryptic chromo somal abnormalities in unexplained mental retardation; a general strategy using hypervariable subtelomeric IDNA polymorphisms. Am J Hum Genet 1993; 53 : 688-701.

\section{7e Symposium international sur la génétique des micro-organismes d'intérêt industriel}

Montréal (Québec) Canada du 26 juin au $1^{\text {er }}$ juillet 1994 notype particulier ne serait alors plus nécessaire pour provoquer la recherche d'un diagnostic. Un travail récent propose, pour cette recherche des cassures submicroscopiques des télomères, l'étude systématique de 29 systèmes polymorphes [9]. Des calculs théoriques permettent à l'auteur de conclure que cette exploration faite en routine pourrait identifier environ $50 \%$ à $70 \%$ des retards mentaux actuellement inexpliqués.

D.L.

1. Weatherall DJ, Higgs IDR, Bunch C, et al. Hemoglobin $\mathrm{H}$ disease and mental retardation - a new syndrome or a remarkable coincidence. N Engl J Med 1981 ; 305 : 607-12. 2. Gibbons RJ, Wilkie AOM, Weatherall IDJ, Higgs IDR. A newly defined $X$ linked mental retardation syndrome associated with $\alpha$ thalassaemia. J Med Genet 1991; 28: 729-33.
- Pour de plus amples renseignements: Secrétariat du Symposium, GIM 94, Conseil national de recherches Canada, Ottawa (Ontario) Canada K1A OR6

Téléphone: (613) 993-9431

Télécopieur: (613) 957-9828.

XX|e Forum des jeunes chercheurs de la Société Française de Biochimie et Biologie Moléculaire

Reims (France) 5-8 juillet 1994

Secrétariat: Pierre Jeannesson, Faculté de Pharmacie, Laboratoire de Biochimie, 51, rue CognacqJay, 51096 Reims Cedex, France. Tél.: 26.05.35.67 - Télécopie : 26.05.37.20

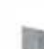

